\title{
Evidence against a separate high affinity binding site on the $\mathbf{P} \mathbf{2} \mathbf{X}_{\mathbf{3}}$ receptor

\author{
Robert Karoly*1, Arpad Mike ${ }^{1}$ Zoltan Gerevich², Peter Illes² and E \\ Sylvester Vizi ${ }^{1}$
}

\author{
Address: ${ }^{1}$ Institute of Experimental Medicine, Budapest, Hungary and ${ }^{2}$ Rudolf Boehm Institute of Pharmacology and Toxicology, Leipzig, Germany \\ Email: Robert Karoly* - karoly@koki.hu \\ * Corresponding author
}

\author{
from I 3th Scientific Symposium of the Austrian Pharmacological Society (APHAR). Joint Meeting with the Austrian Society of Toxicology (ASTOX) and the \\ Hungarian Society for Experimental and Clinical Pharmacology (MFT) \\ Vienna, Austria. 22-24 November 2007 \\ Published: 14 November 2007 \\ BMC Pharmacology 2007, 7(Suppl 2):A38 doi:10.1 I86/147|-2210-7-S2-A38
}

This abstract is available from: http://www.biomedcentral.com//47/-22 10/7/S2/A38

(c) 2007 Karoly et al; licensee BioMed Central Ltd.

It has been proposed that $\mathrm{P} 2 \mathrm{X}_{3}$ receptors possess a unique mechanism of agonist-induced conformational transitions. Recovery from ATP-induced desensitization was found to be very slow; during this period a special agonist binding site was supposed to be formed which should bind the agonist with high affinity and promote desensitization without activation. The authors supposed that this high affinity binding site is absent from non-activated receptors. The theory was supported by an unexpected outcome of an experiment in which a low concentration of agonist was applied at different phases during recovery from desensitization. The inhibition by a low concentration of agonist was stronger when it was applied during the early phase of recovery when more desensitized receptors were present. The authors used different agonists for initial desensitization and for prolonged perfusion at low concentration. We repeated the experiment on HEK 293 cells expressing human $\mathrm{P} 2 \mathrm{X}_{3}$ receptors with the same results. However, when we used the same agonist at both concentrations the inhibition was stronger when the low concentration was applied during the late phase. Simulations revealed that formation of high affinity binding sites does not require any unique mechanism and can be readily described by an allosteric mechanism. Furthermore, they predict that the unexpected phenomenon can only occur when a rapidly dissociating drug is replaced by a slowly dissociating drug on the receptor. 